\title{
Efecto del uso de ensilado seco de salmón en dietas de pollos broiler sobre parámetros productivos y calidad sensorial de la carne
}

\author{
The effects of using dried salmon silage in broiler chicken diets on productive \\ performance and meat sensory quality
}

\author{
C Valenzuela ${ }^{\mathrm{a}}$, F Carvallo ${ }^{\mathrm{b}}$, MS Morales ${ }^{\mathrm{a}}$, P Reyes $^{\mathrm{a}}$ \\ aDepartamento de Fomento de la Producción Animal, Facultad de Ciencias Veterinarias y Pecuarias, Universidad de Chile, Chile. \\ ${ }^{\mathrm{b} C a l i f o r n i a}$ Animal Health and Food Safety Laboratory, San Bernardino, California, USA.
}

\begin{abstract}
SUMMARY
The aim of this study was to determine the effect of using dried salmon silage in broiler diets on performance parameters and meat sensory quality. Dried salmon silage (ESS) was obtained from salmon wastes, processed by acid digestion and co-dried with wheat bran (70:30, respectively). Three hundred Ross 308 broiler chickens were used, and randomly distributed to five feeding groups: control, C (0\% ESS), T1 (4\% ESS), T2 (8\% ESS), T3 (12\% ESS) and T4 (15\% ESS). The chicks were fed for 32 days with these diets, and with control diet only from day 33 to day 42 . The following performance parameters were quantified: mortality percentage $(\% \mathrm{M})$, body weight $(\mathrm{PV})$, daily weight gain $(\mathrm{GPV})$, feed intake (CA), and feed conversion (ECA). After day 42, samples of breast and drumstick meat were obtained for sensory analysis. The diets used were isoproteic and isoenergetic. The use of ESS did not have a significant effect on \% M, PV, GPV, CA and ECA $(\mathrm{P}<0.05)$, and neither did it affect the sensory quality of breast and drumstick meat. In conclusion ESS can be used as an important contribution to broiler feed, and an alternative to protein concentrates that are currently in the market. Additionally, the inclusion of ESS up to $15 \%$ in broiler diets until day 32 does not generate adverse effects on productive performance, and breast and drumstick meat sensory quality.
\end{abstract}

Key words: silage, salmon, broiler.

\section{RESUMEN}

El objetivo de este estudio fue determinar el efecto del uso de ensilado seco de salmón en dietas de pollos broiler sobre parámetros productivos y calidad sensorial de la carne. El ensilaje seco de salmón (ESS) se obtuvo a partir de salmones muertos procesados por digestión ácida y cosecado con afrecho de trigo (70:30, respectivamente). Se utilizaron 300 pollos broiler Ross 308 y se distribuyeron aleatoriamente a cinco grupos de alimentación: control, C (0\% ESS), T1 (4\% ESS), T2 (8\% ESS), T3 (12\% ESS) y T4 (15\% ESS). Los pollos fueron alimentados durante 32 días con estas dietas, y solo con dieta control desde el día 33 al 42 . Se cuantificaron los siguientes parámetros productivos: porcentaje de mortalidad (\%M), peso vivo (PV), ganancia de peso vivo (GPV), consumo de alimento (CA), y eficiencia de conversión alimentaria (ECA). En el día 42 se obtuvieron muestras de carne de pechuga y trutro largo para análisis sensorial. Las dietas utilizadas fueron isoproteicas e isoenergéticas. El uso de ESS no tuvo un efecto significativo en \%M, PV, GPV, CA y ECA ( $<<0,05)$, y tampoco afectó la calidad sensorial de carne de pechuga y trutro largo de pollo. En conclusión, el ESS puede convertirse en una importante contribución a la alimentación de pollos broiler, y una alternativa a los concentrados proteicos que existen actualmente en el mercado, y que su incorporación en dietas de pollos broiler hasta 15\% hasta el día 32 de vida de los animales no genera efectos sobre los parámetros de rendimiento productivo como tampoco en las características sensoriales de la carne de pechuga y trutro largo de pollo.

Palabras clave: ensilado, salmón, pollos broiler.

\section{INTRODUCCIÓN}

Los alimentos concentrados proteicos utilizados en dietas de pollos broiler son considerados de alto costo, siendo las fuentes proteicas más usadas los subproductos de oleaginosas como harina y afrecho de soya, debido a su alto contenido en proteína digestible y equilibrado perfil de aminoácidos (NRC 1994, Liu 2000). Sin embargo, la producción de soya no es suficiente para satisfacer las demandas de la alimentación animal mundial, lo que se

Aceptado: 15.05.2014.

* Casilla 2 correo 15 La Granja, Santiago, Chile; cvalenzuelav@u. uchile.cl. ha visto potenciado en la última década por la elaboración de biocombustibles a partir del poroto de soya (Demirbas 2007, Tomei y Uphamb 2009). Por lo anterior, las tendencias recientes se centran en la investigación de fuentes proteicas alternativas, sustentables y rentables.

Chile es el primer productor de salmón en el hemisferio sur y segundo a nivel mundial, rubro en el que se producen grandes cantidades de desechos desde salmones muertos generando una alta contaminación ambiental y desperdicios de un alimento con muchas bondades nutritivas (Buschmann y col 1996). Una de las metodologías propuestas para el procesamiento de estos desechos ha sido la elaboración de ensilado de pescado (Hassan y Heath 1986, Icaza 1991, Lo y col 1993), este se ha descrito 
como amigable con el medio ambiente, tecnológicamente simple y su procesamiento es más económico que la harina de pescado (Gildberg 1993). La elaboración del ensilado se basa en someter desechos de pescado a fermentación bacteriana, hidrólisis ácida, o enzimática, generando un producto líquido sanitariamente aceptable (Aranson y col 1994). La aplicación de los ensilados líquidos en dietas de animales productivos es compleja, debido a la dificultad de mezcla con dietas que tienen aproximadamente $85 \%$ de materia seca, lo que repercute en su conservación, almacenamiento y la forma de ofrecer las raciones a los animales. Como solución a esta problemática los ensilados líquidos se han mezclado con diferentes tipos de cereales u oleaginosas para facilitar su secado y utilización (Hardy y col 1984, Goddard y Perret 2005), sin embargo, hay pocos estudios en donde se haya investigado el efecto de ensilado de pescado seco incorporado en la dieta de pollos broiler (Hammoumia y col 1998, Santana-Delgado y col 2008), y no hay reportes del uso de ensilado seco a base de desechos de salmón en aves de corral.

Nutricionalmente el ensilado de pescado ha demostrado tener un alto contenido proteico $(\approx 40-55 \%$ base seca) $\mathrm{y}$ un perfil aminoacídico favorable para los animales no rumiantes, con mayores concentraciones de aminoácidos limitantes para las aves de corral como metionina y lisina (Vizcarra-Magaña y col 1999, Vidotti y col 2003) que otras fuentes proteicas (Fernández y col 1994), lo que indica que este producto podría ser estudiado como reemplazo de los concentrados proteicos en proporciones determinadas en las dietas de pollos broiler, para evitar características sensoriales desagradables en la carne como olor y sabor a pescado (Raa y Gildberg 1982, Krogdahl 1985).

Sobre la base de lo descrito anteriormente, el objetivo de este estudio fue determinar el efecto del uso de ensilado seco de salmón en dietas de pollos broiler sobre parámetros productivos y calidad sensorial de la carne.

\section{MATERIAL Y MÉTODOS}

\section{PREPARACIÓN DE LAS DIETAS EXPERIMENTALES}

Ensilado seco de salmón (ESS). El ESS fue proporcionado por la empresa FIORDOAUSTRAL S.A, Chile, procesado desde desechos de mortalidades de varias especies de salmónidos: salmón salar (Salmo salar), coho (Oncorhynchus kisutch) y trucha arcoíris (Oncorhynchus mykiss), tratados por digestión ácida con ácido fórmico al $85 \%$ (40 mL/Kg de pescado) (Oxiquim S.A, Chile), a un rango de temperatura entre $5-15^{\circ} \mathrm{C}$ por 2 semanas, aproximadamente, obteniendo un ensilaje líquido, que posteriormente fue cosecado con salvado de trigo (70 partes de ensilaje de salmón: 30 partes de salvado de trigo) a $100-120^{\circ} \mathrm{C}$, obteniendo el ESS. Al ESS se le determinó el contenido de humedad, proteína cruda (Kjeldhal 945.18, N x 6,25), extracto etéreo, fibra cruda y cenizas (AOAC, 1996).
Dietas experimentales. Se formularon 10 dietas basadas en los requerimientos para pollos broiler (NRC 1994), cinco dietas para el período de alimentación de inicio (0-22 días) y otras cinco para el período final (23-32 días). Las dietas contenían diferentes porcentajes de inclusión de ESS de $0 \%, 4 \%, 8 \%, 12 \%$ y $15 \%$ (cuadro 1 ). A las dietas se les determinó la composición química según la metodología descrita por la AOAC (1996) (cuadro 1).

\section{DISEÑO EXPERIMENTAL}

Este estudio fue aprobado por el Comité de Bioética de la Facultad de Ciencias Veterinarias y Pecuarias de la Universidad de Chile (FAVET), y se realizó en la Unidad Experimental de Producción y Nutrición Avícola de FAVET. Se utilizaron 300 pollos broiler Ross 308 machos, de un día de edad. Los animales se mantuvieron en corrales de piso con camas de viruta de madera, con sistema de alimentación y agua potable ad libitum. La densidad poblacional, condiciones ambientales de temperatura y humedad relativa, y programas de luz/ oscuridad se realizaron según la Guía de Manejo del Pollo de Engorde (COBB 2008).

El estudio tuvo una duración de 42 días. Los animales se pesaron el día 1 y se distribuyeron aleatoriamente a 5 grupos (cada uno con un $\mathrm{N}=60$, en cuadruplicado, separados en 4 subcorrales): Control, C (0\% ESS), T1 (4\% ESS), T2 (8\% ESS), T3 (12\% ESS) y T4 (15\% ESS). Los animales fueron alimentados durante el ensayo con 3 dietas, de inicio (1-22 días), final (23-32 días) (cuadro 1) y de carencia (33-42 días), llamada así por no incluir el ESS, la que correspondió a la dieta control del período final.

\section{PARÁMETROS DE RENDIMIENTO PRODUCTIVO}

Mortalidad (M). La mortalidad se calculó para todo el período de duración del ensayo y se expresó como porcentaje. A todos los animales muertos se les realizó un estudio anatomopatológico por un especialista en la sala de Necropsia del Laboratorio de Patología Animal de FAVET.

Peso vivo $(P V)$. Para calcular el $\mathrm{PV}$ se pesaron los animales individualmente los días 1, 10, 22, 30 y 42 de ensayo. Una hora antes del pesaje las dietas fueron retiradas para todas las aves.

Consumo de alimento (CA). El CA se calculó los días 22 y 42 de ensayo, registrando el alimento ofrecido a los animales, y en cada día de análisis se vaciaron y pesaron los restos de alimento de los comederos, obteniendo el CA por diferencia entre lo ofrecido y no consumido por animal.

Ganancia de peso vivo (GPV) y eficiencia de conversión alimentaria (ECA). Ambos parámetros se calcularon según las ecuaciones 1 y 2 , respectivamente.

$$
G P V=\frac{P f-P i}{42}
$$


Cuadro 1. Ingredientes y composición química de dietas experimentales.

Ingredients and chemical composition of experimental diets.

\begin{tabular}{|c|c|c|c|c|c|c|c|c|c|c|}
\hline \multirow{3}{*}{ Ingredientes (\%) } & \multicolumn{5}{|c|}{ Dieta inicio (1 - 22 días) } & \multicolumn{5}{|c|}{ Dieta final (23 - 32 días) } \\
\hline & \multicolumn{10}{|c|}{ Ensilado seco de salmón (ESS) (\%) } \\
\hline & 0 & 4 & 8 & 12 & 15 & 0 & 4 & 8 & 12 & 15 \\
\hline Maíz & 68,31 & 68,22 & 68,13 & 68,06 & 67,97 & 69,41 & 69,4 & 69,43 & 69,46 & 69,47 \\
\hline Afrecho de soya & 26,02 & 22,27 & 18,52 & 14,77 & 11,97 & 11,72 & 8,93 & 6,13 & 3,34 & 1,02 \\
\hline ESS & 0 & 4 & 8 & 12 & 15 & 0 & 4 & 8 & 12 & 15 \\
\hline Gluten meal & - & - & - & - & - & 12,86 & 12,07 & 11,28 & 10,49 & 10,05 \\
\hline Aceite vegetal & 1 & 1 & 1 & 1 & 1 & 1 & 1 & 1 & 1 & 1 \\
\hline Fosfato dicálcico & 1,75 & 1,60 & 1,46 & 1,31 & 1,2 & 2,07 & 1,74 & 1,41 & 1,07 & 0,86 \\
\hline Conchuela & 1,94 & 1,97 & 2,00 & 2,02 & 2,05 & 1,98 & 1,93 & 1,87 & 1,81 & 1,79 \\
\hline Sal yodada & 0,29 & 0,25 & 0,22 & 0,19 & 0,18 & 0,27 & 0,24 & 0,21 & 0,18 & 0,18 \\
\hline L-Lisina & 0,42 & 0,38 & 0,34 & 0,3 & 0,26 & 0,42 & 0,38 & 0,34 & 0,30 & 0,26 \\
\hline DL-metionina & 0,12 & 0,16 & 0,18 & 0,2 & 0,22 & 0,12 & 0,16 & 0,18 & 0,20 & 0,22 \\
\hline Premix vitaminas ${ }^{1}$ & 0,05 & 0,05 & 0,05 & 0,05 & 0,05 & 0,05 & 0,05 & 0,05 & 0,05 & 0,05 \\
\hline Premix minerales ${ }^{2}$ & 0,05 & 0,05 & 0,05 & 0,05 & 0,05 & 0,05 & 0,05 & 0,05 & 0,05 & 0,05 \\
\hline Coccidiostato $^{3}$ & 0,05 & 0,05 & 0,05 & 0,05 & 0,05 & 0,05 & 0,05 & 0,05 & 0,05 & 0,05 \\
\hline \multicolumn{11}{|c|}{ Composición química en base seca* } \\
\hline $\mathrm{PC}(\%)$ & 23,0 & 23,6 & 23,4 & 23,2 & 22,9 & 20,8 & 20,9 & 21,0 & 20,9 & 20,6 \\
\hline $\mathrm{FC}(\%)$ & 7,1 & 7,4 & 6,5 & 6,9 & 6,9 & 7,4 & 7,4 & 7,5 & 7,8 & 7,8 \\
\hline $\mathrm{EE}(\%)$ & 3,7 & 4,1 & 3,8 & 3,9 & 3,8 & 3,9 & 3,6 & 3,8 & 3,9 & 4,0 \\
\hline $\mathrm{C}(\%)$ & 7,4 & 7,7 & 7,4 & 7,7 & 7,5 & 7,3 & 7,0 & 7,2 & 7,4 & 7,5 \\
\hline ENN (\%) & 58,8 & 57,2 & 58,8 & 58,3 & 58,9 & 60,6 & 61,1 & 60,4 & 60,0 & 60,1 \\
\hline
\end{tabular}

1 Vitaminas, aporte por kilo de dieta: A: 10.000 U.I; D3: 3.500 U.I; E: 50 U.I; K3: 2 mg; B1: 2 mg; B2: 8 mg; B6: 4 mg; B12: 0,015 ng; Niacina: 40 $\mathrm{mg}$; ácido pantoténico: $15 \mathrm{mg}$; biotina: $0,13 \mathrm{mg}$; ácido fólico: $1,5 \mathrm{mg}$; cloruro de colina: $400 \mathrm{mg}$.

2 Minerales, aporte por kilo de dieta: Cu: $8 \mathrm{mg}$; Zn: $80 \mathrm{mg}$; Fe: $80 \mathrm{mg}$; Mn: $100 \mathrm{mg}$; I: $1 \mathrm{mg}$; Se: 0,25 mg.

3 Coccidiostato: $150 \mathrm{mg}$ de lasalocid $/ \mathrm{kg}$.

* Composición determinada por análisis químico proximal. PC: proteína cruda. FC: fibra cruda. EE: extracto etéreo. C: cenizas. ENN: extracto no nitrogenado.

Donde: $P f$ es peso final. $P i$ es peso inicial, y 42 corresponde a los días de duración del ensayo.

$$
E C A=\frac{C A / \text { animal }}{P f}
$$

Donde: $C A$ es consumo de alimento. $P f$ es peso final.

\section{EVALUACIÓN SENSORIAL DE LA CARNE}

El día 42 de ensayo se seleccionaron 8 animales al azar de cada grupo de alimentación, a estos en el día 32 se les retiraron las dietas que incluían el ESS y siguieron siendo alimentados con dieta final control hasta el día 42. Los animales fueron sacrificados por dislocación cervical con posterior sangrado. A partir de las canales se obtuvieron muestras de carne de pechuga y trutro largo, las que fueron guardadas con hueso al vacío y congeladas a $-18^{\circ} \mathrm{C}$. Las muestras se descongelaron en refrigeración $\left(4{ }^{\circ} \mathrm{C}\right)$ el mismo día del análisis sensorial, en ello participó un panel de 12 jueces entrenados. La evaluación se realizó en carne de pollo fileteada ( 3 muestras de $\approx 20$ $\mathrm{g} /$ panelista para cada tratamiento), y cocida $\left(100^{\circ} \mathrm{C} / 10\right.$ $\mathrm{min})$. Se utilizó una prueba discriminativa de aceptabilidad que midió los siguientes atributos: apariencia, intensidad aromática, aroma a pollo, aroma defectuoso, sabor a pollo, sabor defectuoso, textura, persistencia del aroma a pollo, estos se analizaron utilizando una escala no estructurada de 0 a 12 puntos. Las muestras se consideraron aceptables cuando su valor medio fue igual o mayor a 6 puntos. También en el análisis sensorial se incluyó un ítem de observaciones generales para que los jueces anotaran cualquier sensación organoléptica que no estuviera incluida en la prueba discriminativa.

\section{ANÁLISIS ESTADÍSTICO}

Los datos se sometieron a una prueba de normalidad de Shapiro-Wilk. Se calcularon como estadígrafos promedio \pm desviación estándar. Se aplicó un ANOVA y una prueba de Tukey para determinar diferencias significativamente estadísticas $(\mathrm{P}<0,05)$ con el programa Infostat ${ }^{\circledR}$ (Grupo InfoStat, 2002). 


\section{RESULTADOS}

\section{COMPOSICIÓN QUÍMICA DEL ESS Y DIETAS} EXPERIMENTALES

La composición química de ESS en base seca fue la siguiente: humedad $(14,1 \pm 1,0 \%)$, proteína cruda $(50,1$ $\pm 0,8 \%)$, extracto etéreo $(5,7 \pm 2,6 \%)$, fibra cruda $(3,8 \pm$ $0,4 \%)$, cenizas $(10,7 \pm 0,5 \%)$, y extracto libre de nitrógeno $(24,7 \pm 0,9 \%)$. Se destaca el alto contenido proteico del ESS.

No se encontraron grandes diferencias en relación con el contenido de nutrientes entre las dietas experimentales de inicio y final elaboradas con diferentes porcentajes de inclusión de ESS (cuadro 1), siendo isoproteicas e isoenergéticas.

\section{PARÁMETROS DE RENDIMIENTO PRODUCTIVO}

En el cuadro 2 se presentan los parámetros de rendimiento productivo: porcentaje de mortalidad $(\% \mathrm{M})$, peso vivo (PV), ganancia de peso vivo (GPV), consumo de alimento (CA), y eficiencia de conversión alimentaria (ECA) de pollos broiler alimentados con dietas que incluyeron diferentes porcentajes de ESS. El porcentaje de mortalidad obtenido al final del estudio fue bajo y se encontró dentro de los rangos esperables en un manejo productivo comercial. Los hallazgos anatomopatológicos de las aves no mostraron una relación entre la dieta y la causa de la muerte. Según los resultados, los decesos fueron a causa del síndrome de muerte súbita y retraso en el crecimiento.

En cuanto a la variación de PV, durante los 42 días no se observaron diferencias significativas entre los grupos con distintas dietas los días $1,22,32$ y 42 de estudio $(\mathrm{P}>0,05)$.
En el día 10 los animales alimentados con la dieta con $4 \%$ de ESS mostraron un significativo menor PV promedio que los otros animales $(\mathrm{P}<0,05)$, sin embargo, esto no siguió la misma tendencia cuando se realizaron las determinaciones del PV los otros días del estudio (cuadro 2). Respecto de la GPV tampoco se observaron diferencias significativas entre los animales alimentados con las diferentes dietas experimentales $(\mathrm{P}>0,05)$ (cuadro 2$)$.

Los animales alimentados con las dietas de inicio T1, T2, T3 y T4, mostraron un significativo mayor CA que los animales con dieta control $(\mathrm{P}<0,05)$. Al final del estudio se observó algo similar, ya que los animales de los grupos con dietas de finalización T1 y T2 presentaron un significativo aumento del CA, respecto de los animales con dieta final control $(\mathrm{P}<0,05)$, y a su vez los animales alimentados con las dietas T3 y T4 consumieron más alimento que los pertenecientes a los grupos de alimentación T1 y T2 $(\mathrm{P}<0,05)$ (cuadro 2). Aunque al final del estudio el mayor CA de los animales alimentados con dietas que incluyeron el ESS fue más alto que los animales con dieta control, este aumento fue leve entre 90 a $128 \mathrm{~g} /$ pollo, siendo la dieta T4 la que presentó el mayor consumo. Finalmente no se observó un efecto significativo de la inclusión del ESS en las diferentes dietas en la ECA de los animales (P $>0,05)$ (cuadro 2).

\section{EVALUACIÓN SENSORIAL DE LA CARNE DE POLLO}

En el cuadro 3 se presenta la valorización de los atributos analizados en carne de pechuga y trutro largo de pollo por el panel sensorial. Se observó que no hubo diferencias significativas de aceptabilidad en todos los atributos analizados para carne de pechuga y trutro largo de

Cuadro 2. Efecto del ensilado seco de salmón (ESS) a diferentes niveles sobre parámetros de rendimiento productivo de pollos broiler: mortalidad (M\%), peso vivo (PV, g/ave), ganancia de peso vivo (GPV, g/ave/día), consumo de alimento (CA, g/ave), y eficiencia de conversión alimentaria (ECA, g de alimento/g peso vivo).

Effect of dried salmon silage (ESS) at different levels on growth performance of broiler chicks: mortality (M\%), body weight (PV, g/bird), daily weight gain (GPV, g/bird/day), feed intake (CA, g/bird/day), and feed conversion ratio (ECA, g feed/g gain).

\begin{tabular}{lccccc}
\hline \multirow{2}{*}{ Parámetros } & \multicolumn{3}{c}{ Grupos de tratamientos } \\
\cline { 2 - 6 } & C $(0 \%$ ESS $)$ & T1 $(4 \%$ ESS $)$ & T2 $(8 \%$ ESS $)$ & T3 $(12 \%$ ESS $)$ & T4 $(15 \%$ ESS $)$ \\
\hline M (\%) & 1,7 & 0 & 3,3 & 3,3 & 3,3 \\
PV día 1 & $45,2 \pm 3,2^{\mathrm{a}}$ & $46,0 \pm 3,3^{\mathrm{a}}$ & $45,4 \pm 3,1^{\mathrm{a}}$ & $45,0 \pm 3,2^{\mathrm{a}}$ & $46,4 \pm 3,5^{\mathrm{a}}$ \\
PV día 10 & $188,8 \pm 20,9^{\mathrm{a}}$ & $177,5 \pm 27,7^{\mathrm{b}}$ & $198,3 \pm 22,1^{\mathrm{a}}$ & $199,7 \pm 19,5^{\mathrm{a}}$ & $205,3 \pm 19,8^{\mathrm{a}}$ \\
PV día 22 & $495,4 \pm 74,0^{\mathrm{a}}$ & $504,6 \pm 63,0^{\mathrm{a}}$ & $534,4 \pm 70,3^{\mathrm{a}}$ & $521,3 \pm 77,6^{\mathrm{a}}$ & $528,7 \pm 69,9^{\mathrm{a}}$ \\
PV día 30 & $916,6 \pm 169,1^{\mathrm{a}}$ & $919,9 \pm 182,3^{\mathrm{a}}$ & $970,6 \pm 155,9^{\mathrm{a}}$ & $997,7 \pm 161,7^{\mathrm{a}}$ & $985,1 \pm 126,8^{\mathrm{a}}$ \\
PV día 42 & $2026,4 \pm 271,3^{\mathrm{a}}$ & $2075,9 \pm 259,8^{\mathrm{a}}$ & $2132,8 \pm 224,3^{\mathrm{a}}$ & $2136,7 \pm 297,0^{\mathrm{a}}$ & $2105,5 \pm 239,7^{\mathrm{a}}$ \\
GPV & $47,2 \pm 6,4^{\mathrm{a}}$ & $48,3 \pm 5,4^{\mathrm{a}}$ & $49,7 \pm 5,2^{\mathrm{a}}$ & $49,8 \pm 7,0^{\mathrm{a}}$ & $49,0 \pm 5,3^{\mathrm{a}}$ \\
CA día 22 & $1126,7 \pm 14,4^{\mathrm{a}}$ & $1222,9 \pm 18,9^{\mathrm{b}}$ & $1218,9 \pm 15,8^{\mathrm{b}}$ & $1215,8 \pm 10,6^{\mathrm{b}}$ & $1253,9 \pm 18,0^{\mathrm{b}}$ \\
CA día 42 & $6304,4 \pm 41,3^{\mathrm{a}}$ & $6549,3 \pm 29,7^{\mathrm{b}}$ & $6649,7 \pm 11,6^{\mathrm{b}}$ & $6724,3 \pm 34,1^{\mathrm{c}}$ & $6748,7 \pm 34,7^{\mathrm{c}}$ \\
ECA & $3,1 \pm 0,1^{\mathrm{a}}$ & $3,2 \pm 0,2^{\mathrm{a}}$ & $3,1 \pm 0,1^{\mathrm{a}}$ & $3,2 \pm 0,1^{\mathrm{a}}$ & $3,2 \pm 0,1^{\mathrm{a}}$ \\
\hline
\end{tabular}

Letras distintas indican diferencias estadísticamente significativas $(\mathrm{P}<0,05)$. Valores expresados como promedio \pm desviación estándar. 
Cuadro 3. Análisis sensorial de carne de pechuga (P) y trutro largo (TL) de pollos provenientes de diferentes grupos de alimentación con distintos niveles de inclusión de ensilado seco de salmón (ESS).

Sensory analysis of breast (P) and drumstick meat (TL) of chicks from different feeding groups with dried salmon silage (ESS) at different levels.

\begin{tabular}{|c|c|c|c|c|c|c|c|}
\hline \multirow[b]{2}{*}{ Atributos } & \multicolumn{5}{|c|}{ Grupos de tratamientos } & \multicolumn{2}{|c|}{ Significancia } \\
\hline & $\begin{array}{c}\mathrm{C} \\
(0 \% \mathrm{ESS})\end{array}$ & $\begin{array}{c}\mathrm{T} 1 \\
\text { (4\% ESS) }\end{array}$ & $\begin{array}{c}\mathrm{T} 2 \\
(8 \% \mathrm{ESS})\end{array}$ & $\begin{array}{c}\mathrm{T} 3 \\
(12 \% \mathrm{ESS})\end{array}$ & $\begin{array}{c}\mathrm{T} 4 \\
(15 \% \mathrm{ESS})\end{array}$ & Dieta & Jueces \\
\hline $\begin{array}{l}\text { Apariencia }(\mathrm{P})^{1} \\
(\mathrm{TL})^{2}\end{array}$ & $\begin{array}{l}9,9 \pm 1,9 \\
7,3 \pm 3,4\end{array}$ & $\begin{array}{l}9,0 \pm 2,2 \\
8,1 \pm 2,7\end{array}$ & $\begin{array}{l}9,2 \pm 2,6 \\
7,6 \pm 3,2\end{array}$ & $\begin{array}{l}9,6 \pm 1,9 \\
7,6 \pm 3,1\end{array}$ & $\begin{array}{l}8,3 \pm 2,2 \\
7,3 \pm 2,4\end{array}$ & NS & NS \\
\hline Intensidad aromática & $\begin{array}{l}8,1 \pm 2,1 \\
8,8 \pm 2,5\end{array}$ & $\begin{array}{l}8,8 \pm 3,1 \\
9,0 \pm 3,2\end{array}$ & $\begin{array}{l}8,9 \pm 2,8 \\
7,7 \pm 2,3\end{array}$ & $\begin{array}{l}8,1 \pm 2,7 \\
8,1 \pm 3,3\end{array}$ & $\begin{array}{l}8,0 \pm 2,8 \\
8,1 \pm 3,1\end{array}$ & NS & NS \\
\hline Aroma a pollo & $\begin{array}{l}8,9 \pm 2,3 \\
8,9 \pm 2,4\end{array}$ & $\begin{array}{l}9,1 \pm 3,4 \\
8,7 \pm 3,2\end{array}$ & $\begin{array}{l}8,5 \pm 2,9 \\
7,6 \pm 2,3\end{array}$ & $\begin{array}{l}8,8 \pm 2,5 \\
8,5 \pm 2,8\end{array}$ & $\begin{array}{l}8,2 \pm 2,1 \\
8,4 \pm 3,2\end{array}$ & NS & NS \\
\hline Aroma defectuoso & $\begin{array}{l}1,1 \pm 1,3 \\
1,2 \pm 1,7\end{array}$ & $\begin{array}{l}0,8 \pm 0,6 \\
2,0 \pm 1,6\end{array}$ & $\begin{array}{l}1,3 \pm 1,2 \\
2,1 \pm 2,6\end{array}$ & $\begin{array}{l}1,2 \pm 1,6 \\
2,9 \pm 3,7\end{array}$ & $\begin{array}{l}1,7 \pm 1,1 \\
1,9 \pm 1,2\end{array}$ & NS & NS \\
\hline Sabor a pollo & $\begin{array}{l}8,1 \pm 2,7 \\
8,6 \pm 2,7\end{array}$ & $\begin{array}{l}8,8 \pm 2,9 \\
7,9 \pm 3,9\end{array}$ & $\begin{array}{l}7,0 \pm 2,6 \\
6,5 \pm 3,2\end{array}$ & $\begin{array}{l}7,5 \pm 2,9 \\
7,8 \pm 2,9\end{array}$ & $\begin{array}{l}8,3 \pm 2,4 \\
8,5 \pm 3,3\end{array}$ & NS & NS \\
\hline Sabor defectuoso & $\begin{array}{l}1,1 \pm 1,1 \\
2,7 \pm 1,7\end{array}$ & $\begin{array}{l}1,3 \pm 1,3 \\
2,4 \pm 1,1\end{array}$ & $\begin{array}{l}2,2 \pm 1,2 \\
2,2 \pm 1,6\end{array}$ & $\begin{array}{l}1,1 \pm 1,2 \\
1,4 \pm 1,7\end{array}$ & $\begin{array}{l}1,4 \pm 1,5 \\
3,1 \pm 2,8\end{array}$ & NS & NS \\
\hline Textura & $\begin{array}{l}8,3 \pm 2,2 \\
8,5 \pm 2,4\end{array}$ & $\begin{array}{l}8,6 \pm 3,2 \\
7,9 \pm 3,3\end{array}$ & $\begin{array}{l}8,9 \pm 2,2 \\
8,1 \pm 2,9\end{array}$ & $\begin{array}{l}8,5 \pm 2,3 \\
6,7 \pm 2,8\end{array}$ & $\begin{array}{l}8,4 \pm 2,4 \\
6,5 \pm 3,7\end{array}$ & NS & NS \\
\hline Persistencia & $\begin{array}{l}7,5 \pm 2,5 \\
8,1 \pm 3,4\end{array}$ & $\begin{array}{l}7,7 \pm 4,0 \\
7,9 \pm 3,4\end{array}$ & $\begin{array}{l}7,9 \pm 2,3 \\
7,2 \pm 3,4\end{array}$ & $\begin{array}{l}7,5 \pm 3,1 \\
7,7 \pm 3,2\end{array}$ & $\begin{array}{l}7,6 \pm 1,6 \\
7,5 \pm 3,5\end{array}$ & NS & NS \\
\hline
\end{tabular}

Valores promedio \pm desviación estándar de carne de pechuga ${ }^{1}\left(\mathrm{P}\right.$, fila superior) y trutro $\operatorname{largo}^{2}$ (TL, fila inferior) de pollo. *Efecto significativo $(\mathrm{P}<0,05)$. NS $=$ No significativo. Valores expresados como promedio \pm desviación estándar.

pollos alimentados con dietas control y las que incluyeron el ESS en diferentes proporciones $(\mathrm{P}>0,05)$. Además los atributos que hacen referencia a sensaciones organolépticas estándares de la carne de pollo obtuvieron promedios superiores a 7,5 puntos mostrando una alta aceptabilidad por parte de los jueces. Otro aspecto importante a destacar son los bajos promedios obtenidos en los ítems sabor y aroma defectuoso, tanto en carne de pechuga como de trutro largo, demostrando que no hubo percepción de sabor ni olor a pescado en la carne de pollo (cuadro 3), además en el ítem de observaciones ninguno de los jueces apuntaron algo referente a olor o sabor a pescado en las muestras de carne.

\section{DISCUSIÓN}

El contenido de proteína cruda del ESS fue alto y similar al reportado por otros autores para ensilado de sardinas enteras (Sardinella longiceps) elaborado con ácido fórmico y cosecado con salvado de trigo en diferentes proporciones (48,3 a 58,4\% de proteína cruda) (Goddard y Perret 2005), y levemente superior a ensilado hecho a partir de residuos de la pesca del salmón del Atlántico (Salmo salar) procesado por hidrólisis ácida (48\% de proteína cruda) (Llanes y col 2011). Se esperaba que el ESS obtuviera un menor contenido proteico que otros ensilados de pescado, debido a la mezcla de este con el afrecho de trigo, sin embargo este valor se mantuvo alto seguramente por la baja proporción utilizada de este cereal (70:30 ensilado líquido de salmón:afrecho de trigo), como también a su aporte proteico $(\approx 13$ a $15 \%$ de proteína cruda) (NRC 1994). El contenido proteico del ESS fue superior al reportado para afrechos de soya obtenidos a partir de diferentes tratamientos (42 a 47,8\% de proteína cruda) (NRC 1994, Batal y col 2012), y al usado en el presente estudio para la formulación de las dieta de inicio y finalización (45\% proteína cruda).

En relación con el contenido de extracto etéreo del ESS, el valor reportado en este estudio fue inferior al de otros ensilados de pescado líquidos o cosecados con cereales (Vizcarra-Magaña y col 1999, Gerón y col 2007, Llanes y col 2011), a excepción de un ensilaje cosecado con harina de soya (5,29 a 5,61\% de extracto etéreo) (Fagbenro y Jauncey 1993). Como era de esperar, el contenido de fibra cruda fue bajo por ser un alimento de origen animal. Finalmente el contenido de cenizas del ESS fue alto en comparación a otras fuentes proteicas utilizadas en alimentación de pollos broiler (NRC 1994, Batal y col 2012), y similar a otros ensilados elaborados a partir de pescado completo, debido al aporte principalmente de calcio y fósforo de las espinas y huesos de los desechos de salmón (Vizcarra-Magaña y col 1999, Llanes y col 2011).

La concentración proteica del ESS permitió incluirlo en dietas de pollos broiler como reemplazo del afrecho de soya en la etapa de inicio y final, como también del gluten meal en la dieta de finalización, obteniendo dietas 
isoproteicas y de similares contenidos de otros nutrientes como extracto etéreo, fibra cruda y extracto no nitrogenado que hacen comparables los resultados entre los grupos de tratamientos.

En relación con los parámetros de rendimiento productivo al término del estudio no se encontraron diferencias significativas en PV, GPV, y ECA entre los animales alimentados con dietas que incluían distintos niveles de ESS, lo que sugiere que es posible reemplazar en dietas de pollos broiler un cierto porcentaje de los concentrados proteicos utilizados (afrecho de soya y gluten meal) por el ESS, que es de más bajo costo, sin tener repercusiones negativas en el rendimiento productivo. Los valores de PV obtenidos por los animales fueron más bajos que lo esperado para la línea genética, lo que ha sido reportado en otros estudios experimentales con pollos broiler (Ponce y Gernat 2002), y que generó un incremento de la ECA.

El mayor CA de los animales alimentados con dietas que incluían ESS (T1 a T4) generó leves aumentos de PV en estas aves, sin embargo no fueron significativos, y tampoco tuvieron influencia en la ECA. Estos resultados sugieren que los animales no evidenciaron un rechazo a las dietas que incluyeron ESS, e incluso en la medida que aumentó su proporción en el alimento el CA fue mayor. Esto se puede explicar porque la inclusión de ESS es considerada baja si se compara con la de otros trabajos, en donde la adición de ensilado de pescado en dietas de pollos broiler ha alcanzado valores de 30\% (Al-Marzooqi y col 2010) hasta 44\% (Santana-Delgado y col 2008), y en ambos estudios se ha reportado un significativo menor CA por la baja palatabilidad de las dietas.

Relativo a otros reportes en donde se ha incluido ensilado de pescado en dietas de pollos broiler, los resultados han sido controversiales. Algunos autores han obtenido mayores rendimientos productivos en pollos alimentados con dietas que incluían ensilado de pescado, pero otros no han descrito diferencias entre los animales que consumen ensilado y una dieta a base de proteínas de origen vegetal o animal. Johnson y col (1985) informaron que la inclusión de 25, 50 y $100 \mathrm{~g} \mathrm{~kg}^{-1}$ de ensilado de pescado en reemplazo de la harina de soya en dietas de pollos broiler no afectaron significativamente los parámetros de rendimiento productivo de los animales (CA, PV, GPV, ECA, \%M), similar a lo observado en el presente estudio. Santana-Delgado y col 2008 reportaron que no hubo diferencias significativas en el CA de pollos alimentados con una dieta con 0,110 y $220 \mathrm{~g} \mathrm{~kg}^{-1}$ de ensilado de caballa española cosecado con sorgo, en reemplazo de la harina de soya. Sin embargo, al aumentar la inclusión del ensilado a 330 y $440 \mathrm{~g} \mathrm{~kg}^{-1}$, el CA se redujo significativamente, e incluso en estas dos últimas dietas la GPV disminuyó respecto de los tres grupos de alimentación mencionados anteriormente. Al-Marzooqi y col (2010) reportaron una mayor GPV cuando se incluyó ensilado de sardina al 10 y $20 \%$ en remplazo de la harina de soya, debido al mayor consumo de la dieta que contenía ensilado en estas dos proporciones, entre la semana 1 a 3 de vida de los pollos, y también a la mejor calidad aminoacídica de estas dietas. Sin embargo, cuando se utilizó 30\% de ensilado de sardina los animales no mostraron diferencias significativas en la GPV y el CA, en comparación al grupo control (0\% ensilado). En un estudio realizado por Widjastuti y col (2011) indicaron que el remplazo proteico de harina de pescado por ensilado de atún en una proporción de $4 \%$ aumentó el PV final significativamente, en cambio los porcentajes de inclusión de 6 y $8 \%$ no modificaron los PV de los animales respecto del grupo sin ensilado.

Los resultados del análisis sensorial sugieren que la calidad organoléptica de la carne de pechuga y trutro largo de los animales alimentados con las dietas que incluyeron diferentes porcentajes de ESS no mostraron diferencias significativas en comparación con la carne de pollos alimentados con la dieta control, lo que sugiere que la inclusión de hasta $15 \%$ de ESS hasta el día 32 de vida de los pollos no genera cambios sensoriales adversos en la carne de pollo. En varios estudios sensoriales realizados a la carne de pollos alimentados con dietas que incluyen ensilado de pescado se han reportado efectos adversos, por ejemplo, Al-Marzooqi y col (2010) observaron que el panel sensorial calificó con menores puntuaciones los atributos de jugosidad y sabor de la carne de pechuga de pollo en la medida que el porcentaje de inclusión de ensilado de sardina aumentó en la dieta de estos animales (desde 0 a $30 \%$ ), incluso los jueces describieron un sabor desagradable definido como "sospechoso" en carne de pollo alimentados con la dieta que incluía el ensilado al 30\%. Los autores explicaron este hecho debido al alto contenido graso de la sardina, ya que la composición lipídica del pescado en cualquier subproducto es considerado como el factor más crítico que influye en la calidad organoléptica del tejido comestible (Opstvedt 1984). Sin embargo, a pesar que en el presente trabajo se utilizó salmón para generar el ESS que también es considerado como un pescado graso (Toppe y col 2007), no se reportaron características sensoriales adversas en la carne de pollo de los grupos con ESS, posiblemente porque la mezcla del ensilado de salmón con el afrecho de trigo diluyó este efecto, lo que generó un menor contenido de extracto etéreo del ESS, como se describió anteriormente. Además también hay que considerar el efecto de la dieta de carencia, en donde se extrajo el ESS de las formulaciones en los últimos 10 días del engorde de los pollos.

En otro estudio realizado con carne de muslo de pollo, la que por tener más contenido de grasa podría amplificar sabores y olores adversos traspasados por la alimentación, los autores indicaron que la carne de pollos alimentados con la dieta de más alto nivel de ensilado de pescado (100 $\left.\mathrm{g} \mathrm{kg}^{-1}\right)$ y de grasa de pescado $\left(25 \mathrm{~g} \mathrm{~kg}^{-1}\right)$ presentó mal olor y sabor. El mal sabor de la carne fue percibido como el del aceite de pescado (Kjos y col 1999). Ambas investigaciones se contraponen a los resultados del análisis sensorial del presente estudio, debido posiblemente a que 
las dietas que contenían el ESS fueron ofrecidas hasta el día 32 de vida de los animales, y luego se reemplazaron con la dieta control de finalización, justamente para evitar posibles alteraciones organolépticas en la carne de pollo.

Finalmente, a modo de conclusión el incluir hasta $15 \%$ de ESS en dietas de pollos broiler hasta el día 32 de vida no afectó los parámetros productivos ni la calidad sensorial de la carne de pollo, pudiendo utilizarse este producto como reemplazo a los concentrados proteicos usualmente utilizados en alimentación de pollos broiler.

\section{REFERENCIAS}

Al-Marzooqi W, M Al-Farsi, I Kadim, O Mahgoub, J Goddard. 2010. The effect of feeding different levels of sardine fish silage on broiler performance, meat quality and sensory characteristics under closed and open-sided housing systems. Asian-Aust J Anim Sci 23, 1614-1625.

AOAC. 1996. Official Methods of Analysis of AOAC International. $16^{\text {th }}$ ed. AOAC International Gaithersburg, USA.

Aranson, S. 1994. Production of fish silage. In: Martin AM (ed). Fisheries Processing: Biotechnological Applications. Chapman and Hall, London, UK, Pp 245-271.

Batal A, N Dale, M Persia. 2012. Feedstuffs. Ingredient Analysis Table. University of Georgia, Athens, USA.

Buschmann A, D López, A Medina. 1996. A review of the environmental effects and alternative production strategies of marine aquaculture in Chile. Aquacult Eng 15, 397-421.

COBB. 2008. Guía de Manejo del Pollo de Engorde. Cobb-Vantress Philippines Inc., Pasig City, Philippines.

Demirbas A. 2007. Progress and recent trends in biofuels. Prog Energy Combust Sci 33, 1-18.

Fagbenro O, K Jauncey. 1993. Chemical and nutritional quality of stored fermented fish (tilapia) silage. Bioresource Technol 46, 207-211.

Fernández S, S Aoyagi, Y Han, C Parsons, D Baker. 1994. Limiting order of amino acids in corn and soybean meal for growth of the chick. Poultry Sci 73, 1887-1896.

Gildberg A. 1993. Review: enzymic processing of marine raw materials. Proc Biochem 28, 1-15.

Goddard J, J Perret. 2005. Co-drying fish silage for use in aquafeeds. Anim Feed Sci Tech 118, 337-342.

Hammoumia A, M Faid, M El yachiouic, H Amaroucha. 1998. Characterization of fermented fish waste used in feeding trials with broilers. Process Biochem 33, 423-427.

Hardy R, K Shearer, S Spinelli. 1984. The nutritional properties of co-dried fish silage in rainbow trout (Salmo gairdneri) dry diets. Aquaculture 38, 35-44.
Hassan T, J Heath. 1986. Biological fermentation of fish waste for potential use in animal and poultry feeds. Agr Wastes 15, 1-15.

Icaza JF. 1991. Evaluación química proximal, mineral y energética de ensilados de desechos de pescado conservados a distintas temperaturas. Memoria de Título de Médico Veterinario, Facultad de Ciencias Pecuarias y Veterinarias, Universidad de Chile, Santiago, Chile.

Johnson R, R Brown, P Eason, J Sumner. 1985. The nutritional quality of two types of fish silage for broiler chickens. J Sci Food Agric 36, 1051-1065.

Kjos N, A Skrede, M Øverland. 1999. Effects of dietary fish silage and fish fat on growth performance and sensory quality of growingfinishing pigs. Can J Anim Sci 79, 139-147.

Krogdahl Å. 1985. Fish viscera silage as a protein source for poultry. 2. Experiments with meat-type chickens and ducks. Acta Agric Scand 35, 24-32.

Liu K. 2000. Expanding soybean food utilization. Food Technol $54,46-58$

Llanes J, A Bórquez, J Alcaíno, J Toledo. 2011. Physicochemical composition and digestibility of silages from fishery residues in the Atlantic salmon (Salmo salar). Cuban J Agr Sci 45, 417-421.

Lo K, P Liao, Y Gao. 1993. Effects of temperature on silage production from salmon farm mortalities Bioreso Technol 44, 33-37.

NRC, National Research Council. 1994. Nutrient Requirement of Poultry. $9^{\text {th }}$ ed. National Academy of Sciences, Washington, USA.

Opstvedt J. 1984. Fish fats. In: Wiseman J (ed). Fats in Animal Nutrition. Butter-worths, London, UK, Pp 53-82.

Ponce L, A Gernat. 2002. The effect of using different levels of tilapia by-product meal in broiler diets. Poult Sci 81, 1045-1049.

Raa J, A Gildberg. 1982. Fish silage: A review. CRC Crit Rev Food Sci Nutr 16, 383-419.

Santana-Delgado H, E Ávila, A Sotelo. 2008. Preparation of fish silage from Spanish mackerel (Scomberomorus maculates) and its evaluation in broiler diets. Anim Feed Sci Technol 141, 129-140.

Tomei J, P Uphamb. 2009. Argentinean soy-based biodiesel: An introduction to production and impacts. Energ Policy 37, 3890-3898.

Toppe J, S Albrektsen, B Hope, A Aksnes. 2007. Chemical composition, mineral content and amino acid and lipid profiles in bones from various fish species. Comp Biochem Phys B 146, 395-401.

Vidotti R, E Macedo, D Carneiro. 2003. Amino acid composition of processed fish silage using different raw materials. Anim Feed Sci Tech 105, 199-204.

Vizcarra-Magaña L, E Ávila, A Sotelo. 1999. Silage preparation from tuna fish wastes and its nutritional evaluation in broilers. J Sci Food Agric 79, 1915-1922.

Widjastuti T, H Lengkey, R Herianti. 2011. Utilizing waste product of tuna (Thunnus atlanticus) fish silage and its implementation on the meat protein conversion of broiler. Lucrări Ştiinţifice 55, 83-87. 
